\title{
THE EFFECT OF ROTATION ON RGB SURFACE ABUNDANCES
}

\author{
C. CHARBONNEL \\ Laboratoire d'Astrophysique de Toulouse - CNRS UMR 5772 - \\ 14, av.E.Belin, 31400 Toulouse, France
}

\section{Introduction}

Pop II field and globular cluster giant stars (and, to a less extent, Pop I giants) exhibit chemical anomalies which are not predicted by standard stellar evolution theory. Two hypotheses have been proposed to explain these abundance variations, namely the primordial and the evolutionary explanations. A primordial origin for intracluster abundance anomalies (see e.g. Cottrel \& Da Costa 1981) would be related to inhomogeneities in the cluster material due to pollution by a prior generation of massive stars. In the evolutionary hypothesis, abundance variations would be due to nuclear and mixing processes internal to the giant stars themselves. Many good reviews exist on the subject (see e.g. Briley et al. 1994a, Kraft 1994), in which observational evidence supporting both hypotheses are presented. In this conference, Da Costa recalls the most recent observational data, and some excellent poster contributions bring essential clues to the subject.

I will concentrate here on the evolutionary hypothesis. I will first recall the main observations which reveal that an extra-mixing process occurs in low mass stars while they evolve on the red giant branch (RGB), and more precisely between the completion of the standard first dredge-up and the onset of the helium flash. I will then show how rotation-induced mixing can simultaneously account for the observed behavior of carbon isotopic ratios and lithium abundances in low mass giants. This process also avoids large ${ }^{3} \mathrm{He}$ production by low mass stars in the Galaxy. New developments will be proposed.

\section{Observational evidence supporting the evolutionary scenario}

When the stars experience the first dredge-up, the deepening convective envelope brings up to the surface internal matter which was nuclearly-

T.R. Bedding et al. (eds.),

Fundamental Stellar Properties: The Interaction between Observation and Theory, 349-354.

(C) 1997 IAU. Printed in the Netherlands. 
processed during the main-sequence evolution. This leads to modifications of the surface abundances by amounts that depend both on the stellar mass and initial metallicity. In low mass stars, the convective envelope reaches only regions where ${ }^{12} \mathrm{C}$ was processed in favor of ${ }^{13} \mathrm{C}$ and ${ }^{14} \mathrm{~N}$. So basically, the carbon isotopic ratio declines (from 90 to about 20-30), the carbon abundance drops (by about $30 \%$ ) and nitrogen increases (by about $80 \%$ ), but oxygen and all other element abundances remain unchanged. Still according to the standard scenario, the surface abundances then stay unaltered as the convective envelope slowly withdraws during the end of the RGB evolution.

However, observational data on the abundance variations of $\mathrm{C}, \mathrm{N}, \mathrm{O}$, $\mathrm{Na}, \mathrm{Al}$ in evolved stars reveal a different reality.

- Pop II field and globular cluster giants present ${ }^{12} \mathrm{C} /{ }^{13} \mathrm{C}$ ratios lower than 10, even down to the near-equilibrium value of 4 in many cases (Sneden et al. 1986, Smith \& Suntzeff 1989, Brown \& Wallerstein 1989, 1992, Bell et al. 1990, Suntzeff \& Smith 1991, Shetrone et al. 1993, Briley et al., 1994b, 1997).

- In evolved halo stars, the lithium abundance continues to decrease after the completion of the first dredge-up (Pilachowski et al. 1993).

- A continuous decline in carbon abundance with increasing stellar luminosity along the RGB is observed in globular clusters such as M92 (Carbon et al. 1982; Langer et al. 1986), M3 and M13 (Suntzeff 1981), M15 (Trefzger et al. 1983), NGC 6397 (Bell et al. 1979, Briley et al. 1990), NGC 6752 and M4 (Suntzeff \& Smith 1991)

- In some globular clusters (M92, Pilachowski 1988; M15, Sneden et al. 1991; M13, Brown et al. 1991, Kraft et al. 1992; Omega Cen, Paltoglou $\&$ Norris 1989), giants exhibit evidence for $\mathrm{O} \rightarrow \mathrm{N}$ processed material.

- In addition to the $\mathrm{O}$ versus $\mathrm{N}$ anticorrelation, the existence of $\mathrm{Na}$ and $\mathrm{Al}$ vs $\mathrm{N}$ correlations and $\mathrm{Na}$ and $\mathrm{Al}$ vs $\mathrm{O}$ anticorrelations in a large number of globular cluster red giants has been clearly confirmed (Drake et al. 1992, Kraft et al. 1992, 1993, Norris \& Da Costa 1995, Shetrone 1996).

These observations suggest that, while they evolve on the RGB, low mass stars undergo an extra-mixing in the region situated between the hydrogen burning shell (where the material is processed through the CNcycle and possibly the ON-cycle) and the deep convective envelope. This extra-mixing adds to the standard first dredge-up to modify the surface abundances. Observations of ${ }^{12} \mathrm{C} /{ }^{13} \mathrm{C}$ ratios in $\mathrm{M} 67$ evolved stars (Gilroy \& Brown 1991) strongly suggest that the extra-mixing process is only efficient when the hydrogen burning shell has crossed the discontinuity in molecular weight built by the convective envelope during the first dredgeup (Charbonnel 1994). Before this evolutionary point, the mean molecular 
weight gradient probably acts as a barrier to the mixing in the radiative zone. Above this point, no gradient of molecular weight exists anymore above the hydrogen burning shell, and extra-mixing is free to act.

\section{Rotation-induced mixing on the red giant branch}

Different mixing processes were proposed to explain the abundance anomalies in evolved stars. Sweigart \& Mengel (1979) suggested that meridional circulation induced by stellar rotation on the RGB could lead to the low ${ }^{12} \mathrm{C} /{ }^{13} \mathrm{C}$ ratios observed in field giants. More recently, Charbonnel (1995), Wasserburg et al. (1995), and Denissenkov \& Weiss (1996) reconsidered this idea in order to explain the carbon and oxygen isotope problems on the RGB and AGB. Moreover, if $\mathrm{Na}$ and $\mathrm{Al}$ were produced in the $\mathrm{CN}$ - and $\mathrm{ON}$ - processed region, the deep mixing scenario may also explain $\mathrm{Na}, \mathrm{Mg}$ and $\mathrm{Al}$ anomalies (Denissenkov \& Denissenkova 1990, Langer et al. 1993; Langer \& Hoffman 1995, Denissenkov \& Weiss 1996, Cavallo et al. 1996).

Rotation-induced mixing seems to be a very promising candidate. We investigated the influence of such a process on the RGB (Charbonnel 1995), by taking into account the most recent progress in the description of the transport of chemicals and angular momentum in stellar interiors : We used Zahn's (1992) consistent theory which describes the interaction between meridional circulation and turbulence induced by rotation. In this framework, the global effect of advection moderated by horizontal turbulence can be treated as a diffusion process. In our context, four important points must be emphasized : 1 . The resulting mixing of chemicals in stellar radiative regions is mainly determined by the loss of angular momentum via a stellar wind. 2. Even in the absence of such mass loss, some mixing can take place wherever the rotation profile presents steep vertical gradients. 3. Additional mixing is expected near nuclear burning shells. 4. Due to the stabilizing effect of the composition gradients, the mixing will be efficient on the RGB only when the hydrogen-burning-shell will have crossed the chemical discontinuity created by the convective envelope during the first dredge-up. Since all these conditions are expected to be fulfilled during the non-homologous evolution of low mass stars on the RGB, we suggested that this process could be responsible for the extra-mixing we are looking for.

We estimated the effect of rotation-induced mixing on some surface abundances. Stellar evolutionary models were computed with the Toulouse code, a version of the Geneva stellar evolution code in which we have introduced the numerical method described in Charbonnel et al. (1992) to solve the diffusion equation. We restricted our study to the case where the stars undergo a moderate wind (see Zahn 1992).

Figures 1 and 2 show the influence of rotation-induced mixing on the 

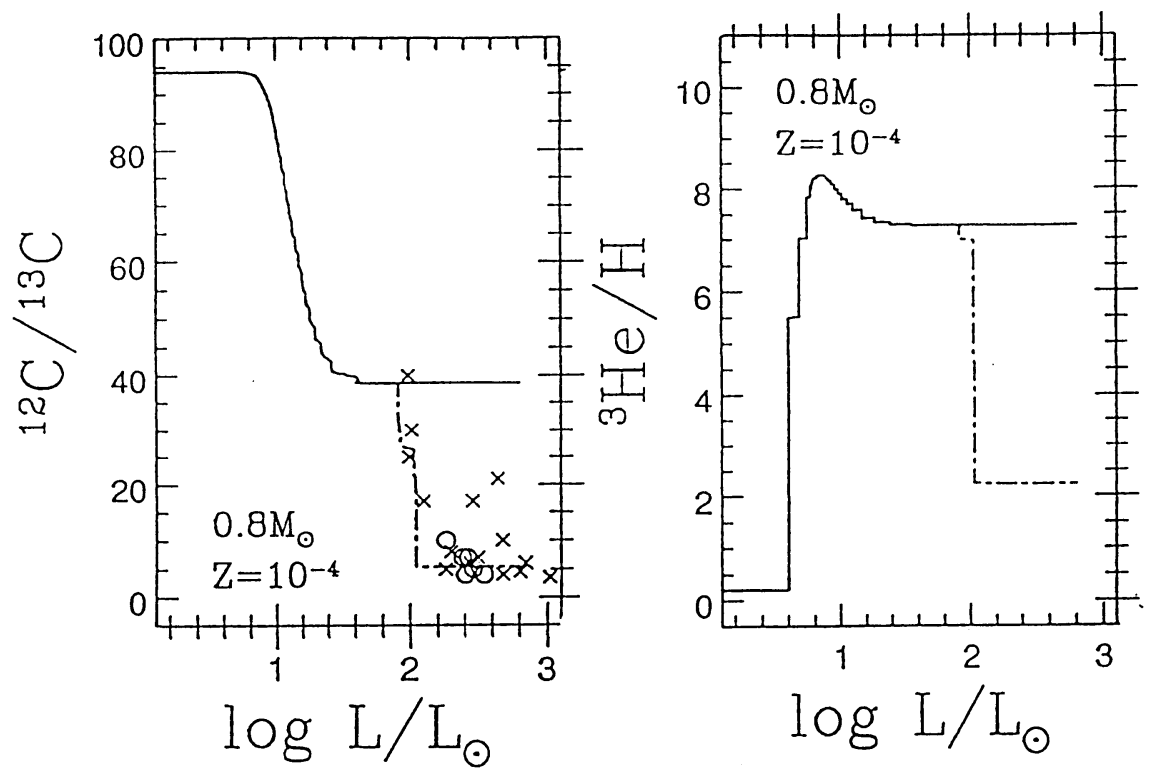

Figure 1. Theoretical behavior of ${ }^{12} \mathrm{C} /{ }^{13} \mathrm{C}$ and of ${ }^{3} \mathrm{He} / \mathrm{H}$ (in units of $10^{-4}$ as a function of luminosity, for standard evolution (solid lines) and for the evolution including extra-mixing (dashed-dotted lines). Observations of the carbon isotopic ratio in field Population II (crosses; Sneden et al. 1986) and globular cluster M4 (circles; Smith \& Suntzeff 1989) giant stars

surface values of ${ }^{12} \mathrm{C} /{ }^{13} \mathrm{C}$ ratio and ${ }^{7} \mathrm{Li}$ abundance in Pop II giants. In the standard case, the post-dilution values of the carbon isotopic ratio and of the ${ }^{7} \mathrm{Li}$ abundance remain constant and are substantially higher than observed in the most evolved RGB stars. However, when extra-mixing begins to act, the ${ }^{12} \mathrm{C} /{ }^{13} \mathrm{C}$ rapidly drops. The observed behavior of ${ }^{12} \mathrm{C} /{ }^{13} \mathrm{C}$ is well reproduced, and one reaches the low values currently observed in globular cluster giants, namely $3-8$. Simultaneously, when ${ }^{7} \mathrm{Li}$ diffuses, it rapidly reaches the region where it is burned by proton capture. Due to rotation-induced mixing on the RGB, the surface abundance of lithium rapidly decreases down to the very low values observed in the halo giants. In addition, ${ }^{3} \mathrm{He}$ also reaches the region where it is nuclearly burned by the ${ }^{3} \mathrm{He}(\alpha, \gamma){ }^{7} \mathrm{Be}$ reaction. This leads to a rapid decrease of the surface value of ${ }^{3} \mathrm{He} / \mathrm{H}$, confirming the predictions by Hogan (1995). This result strongly modifies the actual contribution of low mass stars to the galactic evolution of ${ }^{3} \mathrm{He}$. 


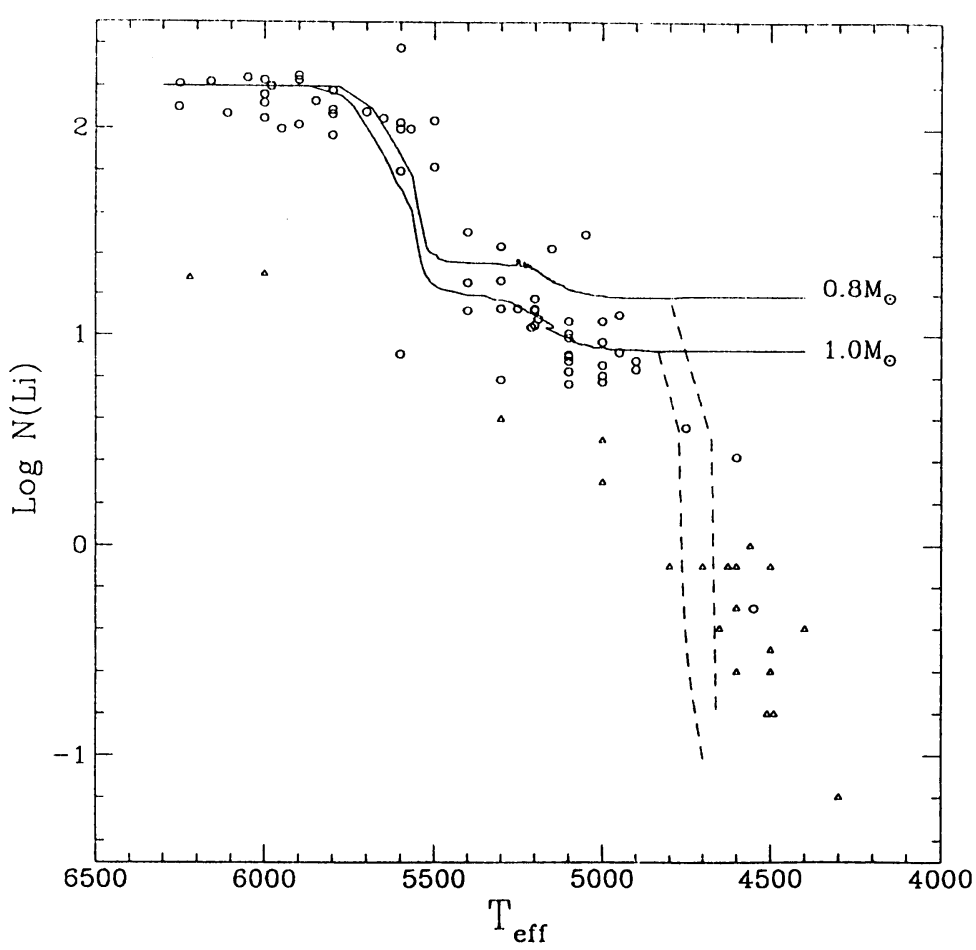

Figure 2. Theoretical behavior of the lithium abundance as a function of $\mathrm{T}_{\text {eff }}$, for standard evolution (solid lines) and for the evolution including extra-mixing (dashed-dotted lines), for 0.8 and $1 \mathrm{M}_{\odot}$ models computed with $\mathrm{Z}=10^{-4}$. The very low lithium abundances observed in the most evolved stars of the sample (halo giant stars from Pilachowski et al. (1993); open circles for real lithium detection, open triangles for upper limits) can only be reproduced when rotation-induced mixing is taken into account

\section{Future developments}

Preliminary results indicate that a realistic physical process, rotation-induced mixing, can simultaneously account for the observed behavior of carbon isotopic ratios and for the lithium abundances in Population II low mass giants. It also avoids large ${ }^{3} \mathrm{He}$ production by low mass stars in the Galaxy.

Detailed simulations, with a consistent treatment of the transport of matter and angular momentum, have now to be carried out for different stellar masses, initial metallicities, mass loss and rotational histories. The impact of this process on other chemical anomalies on the RGB (C $\downarrow, \mathrm{Na} \uparrow$, $\mathrm{O} \downarrow, \mathrm{Al} \uparrow, \mathrm{Mg} \mathrm{O} \downarrow)$ and on the precise yields of ${ }^{3} \mathrm{He}$ has to be investigated in details. 


\section{References}

Bell R.A., Briley M.M., Smith G.H., 1990, AJ 100, 187

Bell R.A., Dickens R.J., Gustafsson B., 1979, ApJ 229, 604

Briley M.M., Bell R.A., Hesser J.E., Smith G.H., 1994a, Can. J. Phys. 72,772

Briley M.M., Bell R.A., Hoban S., Dickens R.J., 1990, ApJ 359, 307

Briley M.M., Smith V.V., King J., Lambert D.L., 1997, AJ 113, 1

Briley M.M., Smith V.V., Lambert D.L., 1994b, ApJ 429, L119

Brown J.A., Wallerstein G., 1989, AJ 98, 1643

Brown J.A., Wallerstein G., 1992, AJ 104, 1818

Brown J.A., Wallerstein G., Oke J.B., 1991 AJ 101, 1693

Carbon D.F., Langer G.E., Butler D., Kraft R.P., Trefzger C.F., Suntzeff N.B., Kemper E., Romanishin W., 1982, ApJS 49, 207

Cavallo R.M., Sweigart A.V., Bell R.A., 1996, preprint

Charbonnel C., 1994, A\&A 282, 811

Charbonnel C., 1995, ApJ 453, L41

Charbonnel C., Vauclair S., Zahn J.P., 1992, A\&A 255, 191

Cottrell P.S., Da Costa G.S., 1981, ApJ 245, L79

Denissenkov P.A., Denissenkova S.N., 1990, SvA Lett. 16, 275

Denissenkov P.A., Weiss A., 1996, A\&A 308, 773

Drake J.J., Smith V.V., Suntzeff N.B., 1992, ApJ 395, L95

Gilroy K. K., Brown J.A., 1991, ApJ 371, 578

Hogan C.J., 1995, ApJ 441, L17

Kraft R.P., 1994, PASP 106, 553

Kraft R.P., Sneden C., Langer G.E., Prosser C.F., 1992, AJ 104, 645

Kraft R.P., Sneden C., Langer G.E., Shetrone M.D., 1993, AJ 106, 1490

Langer G.E., Hoffman R., 1995, PASP 107, 1177

Langer G.E., Hoffman R., Sneden C., 1993, PASP 105, 301

Langer G.E., Kraft R.P., Friel E., Oke J.B., 1986, PASP 97, 373

Norris J.E., Da Costa G.S., 1995, ApJ 441, L81

Paltoglou G., Norris J., 1989, ApJ 336, 185

Pilachowski C.A., 1988, ApJ 326, L57

Pilachowski C.A., Sneden C., Booth J., 1993, ApJ 407, 713

Smith V.V., Suntzeff N.B., 1989, AJ 97, 1699

Sneden C., Kraft R.P., Prosser C.F., Langer G.E., 1991, AJ 102, 2001

Sneden C., Pilachowski C.A., VandenBerg D.A., 1986, ApJ 311, 826

Shetrone M.D., 1996, AJ

Shetrone M.D., Sneden C., Pilachowski C.A., 1993, PASP 195, 337

Suntzeff N.B., 1981, ApJS 47,1

Suntzeff N.B., Smith V.V., 1991 ApJ 381, 160

Sweigart A. V., Mengel J. G., 1979, ApJ 229, 624

Trefzger C.F., Carbon D., Langer G.E., Suntzeff N.B., Kraft R.P., 1983, ApJ 266, 144

Wasserburg G. J., Boothroyd A. I., Sackmann I. J. 1995, ApJ 447, L37

Zahn J.P., 1992, A\&A 265, 115 